\title{
Microwave ferrites for phase shifters
}

\author{
PRAN KISHAN \\ Solid State Physics Laboratory, Delhi 110054, India
}

\begin{abstract}
The electronically steerable phased array radars (PARs) are finding extensive use in military, communication and civilian applications. A PAR system typically comprises a regular arrangement of a large number of phase controlled radiating elements. Ferrite-based phase shifters in various designs are employed in these systems operating at frequencies above $5 \mathrm{GHz}$ due to their superior power rating and insertion loss characteristics. Under DRDO's integrated missile programme considerable success has been achieved in the country on the development of ferrite materials, phase shifters and array system.

The work done at SSPL on the development of ferrite materials for both X-and C-band phase shifters is described. The effect of various substituents in the chemical composition of the lithium-titanium ferrites and of the process parameters to evolve the grades with required magnetic, electrical and microstructural properties are discussed. The investigations carried out to enhance optimally the r.f. power handling capability of the material for meeting the desired specifications of the C-band phase shifter are also given.
\end{abstract}

Keywords. Ferrites; phase shifters; phase array radars.

\section{Introduction}

Taking into consideration the performance and economics of the modern military radar systems the phased array radars (PARs) are described as the expensive ultimate technology (Bullock 1982; Curtis 1986). In a conventional radar the concave dish antenna ceaselessly keeps turning, radiating the microwave beam in space in search of distant objects. In PAR the microwave beam scans the sky without any mechanical movement of the radar. A PAR essentially consists of an array of small identical radiating elements capable of transmitting and receiving signals individually. The simultaneous radiation of energy takes place at the same frequency from a number of elements and the resulting beam is formed as a consequence of their interference. Each unit consists of a phase shifter and a radiating element. The beam steering is carried out by electronically controlling the measure of the relation between the originating wavefronts from each element. A flat regular arrangement of elements, consisting of a large number of units works as a multifunction antenna. It can perform jobs such as surveillance, tracking, illuminate and engage several targets and provide guidance. Formation of multiple beams with equal or unequal beam widths and simultaneous switching of the multiple beams becomes possible by grouping the antenna elements in subarrays and combining the subarrays in different ways. In this way a number of targets are handled simultaneously by the radar. The PATRIQT of gulf war fame, PAVE PAWS, COBRA DANE, AEGIS are well known names of phased array radars being employed in U.S. defence systems. RAJENDRA radar, developed in India for the integrated missile programme of DRDO Project AKASH is the latest addition to the list of PARs. With rapid advances in technology the application of phased array systems are being extended for satellite and ground mobile vehicular communication systems. Among the various types of phase shifter elements available for this purpose their choice is decided by the application of the array. 


\section{Phase shifters}

At microwave frequency ranges phase shifters are successfully employed in practical systems for switching and control of signals. Phased array antennas are the major application area of phase shifters. An array for single axis scanning uses tens to hundreds of these units while a two-axis scanning array needs a few thousands of them. The phase shifters are mostly designed with integrated receiving and radiating antenna elements for use in array systems.

The phase control elements are available in various configurations - both mechanical and electronic (Koul and Bhat 1991). The mechanical phase shifters are rugged and simple to fabricate but not suitable for array systems. The electronic phase shifters provide inertialess phase change in minimal time, and are classified as ferrite, semiconductor, active FET or bulk semiconductor devices. The ferrite and semiconductor devices have pushed the other known phase shifting techniques like slow-wave tubes and bulk plasma into the background.

The ferrite phase shifters have been developed in both reciprocal and non-reciprocal types operating in analog or digital mode. Besides waveguide geometry other configurations like coaxial line, stripline and microstripline have also been employed. In semiconductor phase shifters the electronic control elements are junction devices. The $p$ - $i-n$ diode and GaAs FET are used as electronic switches in digital planar hybrid and monolithic phase shifters respectively. Variable reactance characteristics of varactor diodes are made use of in analog type phase shifters in MIC configuration. The active FET phase shifters provide RF signal amplification along with phase shift in both analog and digital modes. Bulk semiconductor elements, based on dynamic control of conductive properties of electron-hole plasma in semiconductor materials, is reported to be active practical device at $\mathrm{mm}$-wave frequencies. The most recent advancement in this field is the trans-receive (T/R) module where phase shifter, switch and amplifier are united on a GaAs chip by MMIC technology. Ultimately it may include a microprocessor to perform various controls.

Both ferrite and semiconductor devices are characterized by their special features and constraints. Comparison between ferrite phase shifters and commonly used semiconductor phase shifters ( $p-i-n$ diode and FET) can be briefly described. Ferrite phase shifters are generally favoured at frequencies above $5 \mathrm{GHz}$, provide low losses, higher power rating, greater precision of operation and have excellent radiation hardness. On the other hand the semiconductor phase shifters have fast switching rates, require small switching power and being tiny elements have very small weight and size.

\subsection{Types of ferrite phase shifters}

Several types of microwave ferrite phase shifters have been investigated since 1950 . The following three have emerged from the viewpoint of their utility in the system.

(i) Axial toroidal phase shifter: This non-reciprocal phase shifter essentially consists of a ferrite toroid centred axially along a rectangular waveguide (Truehaft and Silber 1958). The magnetization state of the ferrite is switched between two oppositely directed magnetization states of equal magnitude on the hysteresis curve of the material to obtain the phase shift. For this purpose a switching wire threading the toroid is used to change the magnetization state (figure 1a). For improving the efficiency the version 
with dielectric filled toroid window was developed (figure 1b). Twin toroid with dielectric spacer is the currently preferred geometry (figure 1c). High dielectric material is employed for concentrating the microwave field near the central region spanning the broad wall of the rectangular waveguide.

(ii) Dual mode phase shifter: Figure 2 describes the schematic version of a dual mode type phase shifter. It consists of two non-reciprocal phase shift channels. Signals are allowed to proceed from left to right in one channel and in opposite direction in the other undergoing equal phase shift, say $\phi_{1}$. The two channels can be combined by using a dual mode waveguide wherein the same space is used to accommodate the two non-reciprocal phase shifters operating on the two senses of circular polarization. Non-reciprocal circular polarizers provide the functions of two circulators at the input and output ends. The phasor section is simply a variably Faraday rotator. It is realized in both circular and rectangular cross sections (Boyd 1970).

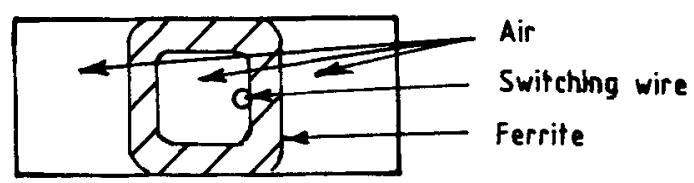

(a)

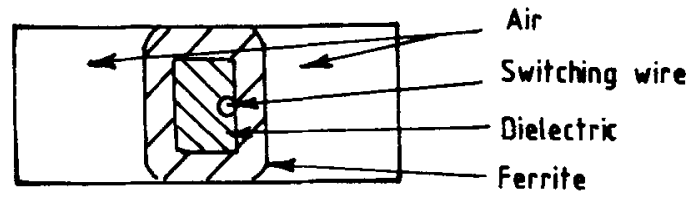

(b)

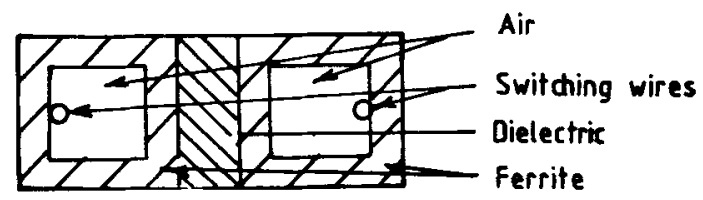

(c)

Figure 1. Configuration of axial toroidal phase shifter.

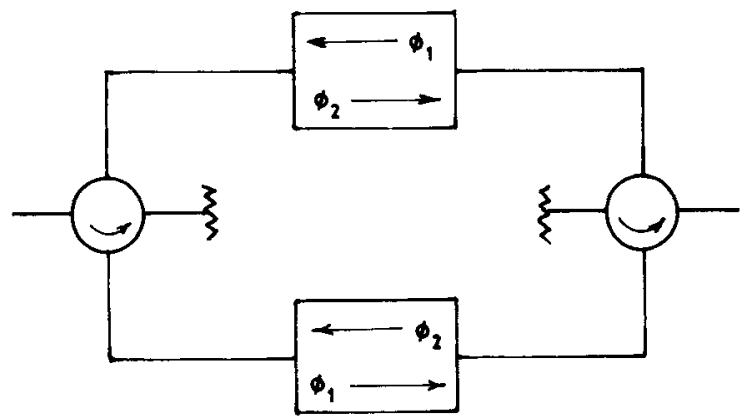

Figure 2. Basic concept of dual mode phase shifter. 


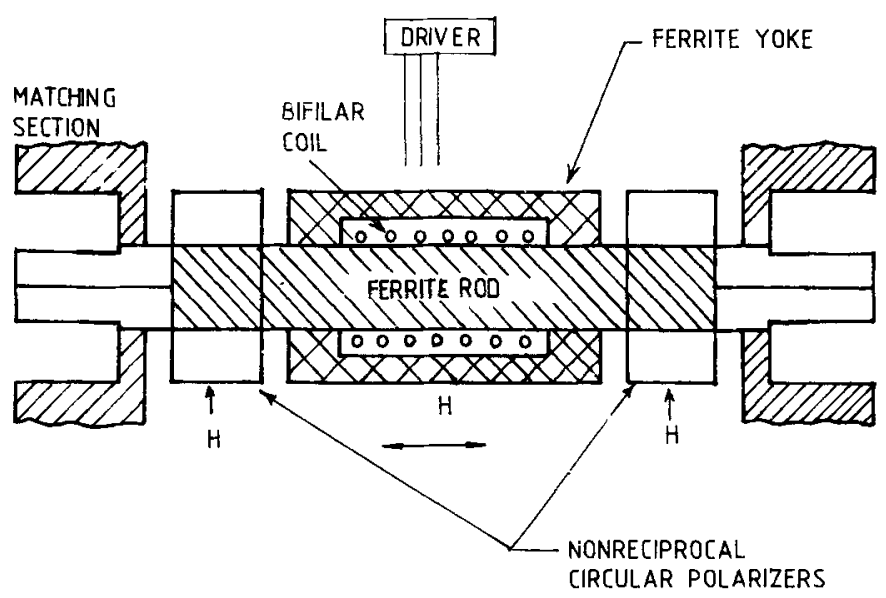

Figure 3. Configuration of a dual mode phase shifter.

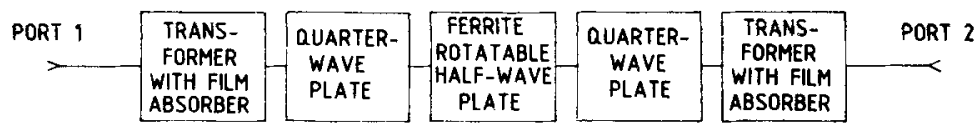

Figure 4. Block diagram of a rotary field phase shifter.

A typical dual mode phase shifter comprises of a ferrite rod metallized to form a completely filled waveguide geometry. The axially magnetized section of the ferrite rod is fitted with an external ferrite yoke to provide the close magnetic path. It is latched to various magnetization states by means of a coil driven by a driver unit to perform the phase shifting operation. The schematic construction of this type of phase shifter is shown in figure 3. Dual mode phase shifter with polarization ability has also been reported (Boyd 1990).

(iii) Rotary field phase shifter: The phasor section in this type of phase shifter is a rotatable half wave plate as shown in figure 4 . The phase shift angle is proportional to twice the angle of rotation of the half waveplate (Fox 1947). The action of rotating half wave plate is generated by means of a multislot stator surrounding the ferrite rod. The winding on the stator are driven by current pulse from a driver unit (Boyd 1976). Development of latching type rotary field phase shifter has been reported recently.

Table 1 depicts the performance characteristics of these three types of phase shifters. The various parameters indicate that axial toroid type is best choice for wide band fast switching application operating at low frequencies. Dual mode is preferred for high frequency short range, two axis scanning radar. The high accuracy of the rotary field type makes it suitable for low side-lobe single axis scanning radar.

\section{Microwave ferrites}

A large number of microwave devices like isolators, circulators, phase-shifters, limiters, tunable filters etc. employ ferrite materials to control the transmission of energy and 
Table 1. Performance characteristics of three types of phase shifters.

\begin{tabular}{|c|c|c|c|}
\hline Parameters & Axial toroidal & Dual mode & Rotary-field \\
\hline Phase shift range & $0-360^{\prime}$ & $0-360^{\circ}$ & Unlimited \\
\hline Insertion loss (dB) & Moderate $(<1)$ & Moderate $(<1)$ & Low $(<0.5)$ \\
\hline Band width & Wide (Octave) & Moderate $(20 \%)$ & Limited $(10 \%)$ \\
\hline $\begin{array}{l}\text { Switching time } \\
\quad(\mu \mathrm{sec})\end{array}$ & Low $(<10)$ & Moderate $(<150)$ & Moderate $(<250)$ \\
\hline $\begin{array}{c}\text { Phase accuracy } \\
\text { (RMS error) }\end{array}$ & Moderate $\left(<4^{\circ}\right)$ & Moderate $\left(<4^{\circ}\right)$ & High $\left(<1^{\circ}\right)$ \\
\hline Power handling & Moderate & Moderate & High \\
\hline Transverse size & Small & Medium & Large \\
\hline Cost & Moderate & Low & Moderate \\
\hline
\end{tabular}

perform a number of functions in microwave systems. The properties of ferrites which make these materials useful for applications at high frequencies are: (i) high resistivity - the microwave frequency electromagnetic waves can penetrate these materials and propagate with low dielectric loss due to their good insulator properties and (ii) the permeability of ferrite can be varied by the application of magnetic field.

The ferrites are compounds of metal oxides containing magnetic ions arranged in a manner to produce spontaneous magnetization. The ferrites belonging to spinel and garnet families manifest the necessary magnetic and dielectric properties for microwave devices. The magnetization of ferrite arises due to ferrimagnetic interaction between the magnetic ions occupying the sublattices of the crystal structure.

Spinel $\mathrm{MeO}-\mathrm{Fe}_{2} \mathrm{O}_{3}$ where $\mathrm{Me}^{2+}=\mathrm{Mg}^{2+}, \mathrm{Ni}^{2+}, \mathrm{Fe}^{2+},\left(\mathrm{Li}_{0 \cdot 5}^{1+} \mathrm{Fe}_{0 \cdot 5}^{3+}\right)$ etc.

Garnet $3 \mathrm{R}_{2} \mathrm{O}_{3}-5 \mathrm{Fe}_{2} \mathrm{O}_{3}$ where $\mathrm{R}^{3+}=$ Rare-earth ion or $\mathrm{Y}^{3+}$.

A variety of metal ions can be substituted in these structures and this has made it possible to prepare a number of families of ferrite materials for applications in diverse components in the different frequency regions. The substituent metal ion has often a preference for occupying a particular site in the crystal structure and thereby help in the selection of material composition.

Most microwave ferrites employed in practical microwave components are polycrystalline. Single crystals and thin films also find applications in many devices. In the following text we shall consider only the ceramic materials as these are of interest to us for use in phase shifters.

\subsection{Interaction of ferrites with microwaves}

Operation of ferrite device at microwave frequencies can be understood by the interaction of the magnetization of the ferrite with the magnetic vector of incident wave. Placing of a ferrite sample in rf field coupled at right angle to a dc magnetic field gives rise to effects like isolation and phase-shift necessary for microwave devices. The microwave properties of the material under such conditions are given by

$$
\mathbf{B}=\mu \mathbf{H},
$$


where $\mu$ is described by Polder tensor (Rosenbaum 1983) and relates microwave induction $\mathbf{B}$ to microwave field intensity $\mathbf{H}$. For the case of small magnetic losses the permeability components are complex in nature

$$
\mu=\mu^{\prime}-j \mu^{\prime \prime}
$$

For a fixed frequency plane wave $\mu$ as a function of field is shown in figure 5 . The individual permeabilities for two circularly polarized components of plane wave are designated by + ve and - ve signs. With proper combination of field and frequency for one sense of circular polarization gyromagnetic resonance $\left(\mu_{+}^{\prime \prime}\right)$ and dispersion $\left(\mu_{+}^{\prime}\right)$ effects are observed. For the opposite sense of polarization $\left(\mu_{-}^{\prime}, \mu_{-}^{\prime \prime}\right)$ the response is almost flat. This non-reciprocal behaviour is exploited for microwave device operation.

A circularly polarized wave propagating in one direction will be absorbed because of $\mu_{+}^{\prime \prime}$ resonance peak but a wave of opposite direction will pass unattenuated. In this way ferrite device acts as a resonance isolator. Propagation velocity of the wave can be varied by exploiting the dispersive behaviour of $\mu_{+}^{\prime}$ thus resulting in rotation of the magnetic vector (Faraday rotation) or a shift in phase. In short, it is the change in permeability achieved by varying the magnitude and direction of the magnetic field which causes the change in propagation constant and leads to the intended effects desired in the operation of ferrite devices. The devices are constructed in many ways by taking advantage of the different possible configurations (Dionne 1975).

\subsection{Latching operation}

Another important property of ferrite is its hysteresis loop behaviour. In a phase shifter device the ferrite is used in closed magnetic path in the form of a toroid (axial toroid type) or by providing an external yoke (in dual mode type). In such an arrangement the magnetization can be driven to different states around the hysteresis loop path of the solid or composite toroid by applying a current pulse from a driver unit. The magnetization then latches on to a remanent value. The phase changes are affected by varying the magnetization states. Point $\mathrm{A}^{\prime}$ or $\mathrm{B}^{\prime}$ (figure 6) corresponding to saturation condition on the hysteresis loop ( $A$ or $B$ ) is chosen as the reference reset point. The change in flux is then carefully set to the desired states like $C^{\prime}$ or $\mathrm{D}^{\prime}$. In this way the propagation constant can be incremented in discrete steps. It may be seen that here remanent magnetization replaces the dc applied field. Ferrite materials with rectangu-

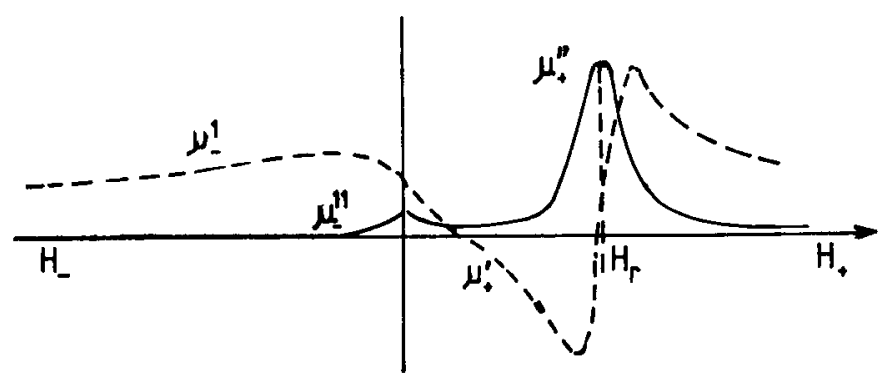

Figure 5. Magnetic permeability components as a function of magnetic field. 
lar nature of the hysteresis loop are required for these devices. The desired properties are high remanence ratio $\left(R=4 \pi M_{\mathrm{r}} / 4 \pi M_{\mathrm{s}}\right)$ and low coercive force $\left(H_{\mathrm{c}}\right)$.

Both toroidal and dual mode phase shifters are designated as latching type while the rotary field does not depend upon the remanent magnetization.

\subsection{High power effect}

The peak power handling capability of the ferrite material must be taken into consideration for application in medium and high power devices. The increase in incident power beyond a critical level causes the loss occurring in the material to rise dramatically due to onset of nonlinear effects (figure 7). The corresponding threshold is

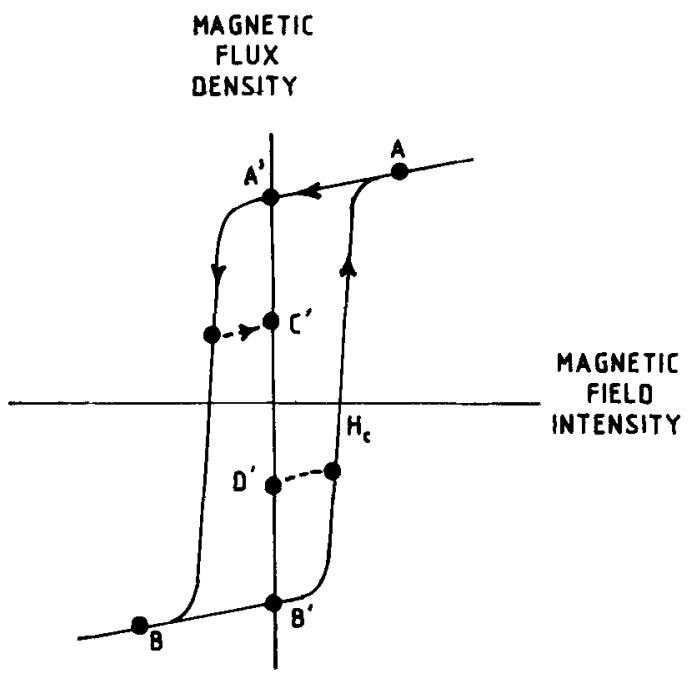

Figure 6. Typical ferrite hysteresis loop.

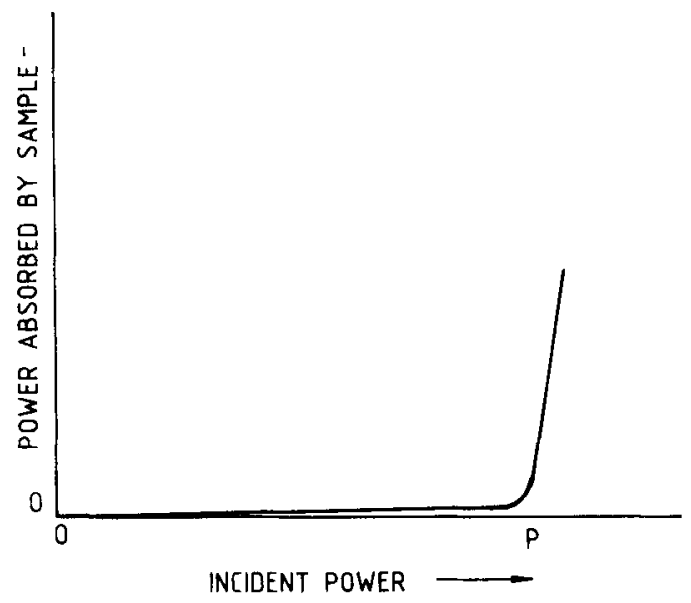

Figure 7. Power absorption as a function of incident power (in a parallel-pumping experiment). 
connected to the existence of a critical microwave field $\left(h_{\mathrm{c}}\right)$ which puts a limit on the power rating of a microwave device. For ferrites the material parameter $\Delta K_{\mathrm{k}}$ (the spinwave linewidth) is directly related to $h_{\mathrm{c}}$ and is used to describe the power handling capability of the material. $\Delta H_{\mathrm{k}}$ value is tailored for the material by incorporating certain fast relaxing ions in the composition and also by microstructural control.

\subsection{Ferrite materials for phase shifters}

The performance of a phase shifter is based on the properties of the ferrite material used in its fabrication. The device parameters such as frequency and bandwidth of operation, phase shift range, insertion loss, phase accuracy, switching time and power, power handling capability besides cost and weight are taken into consideration in deciding the type of phase shifter needed and subsequently the choice of ferrite material.

Amongst the various ferrite materials used in practical phase-shifters the following three families have been favoured: (i) magnesium-manganese ferrites, (ii) lithiumtitanium ferrites and (iii) yttrium-iron garnets.

Materials belonging to first two types possess excellent rectangular loop properties and are considered suitable for latching type of phase shifters. However, MgMn ferrites suffer from several drawbacks like limited $4 \pi M_{\mathrm{s}}$ range (up to $3000 \mathrm{G}$ ), low Curie temperatures and low power rating. The garnets are costly materials having a very limited $4 \pi M_{\mathrm{s}}$ range (up to $1800 \mathrm{G}$ ) with moderate rectangularity of the hysteresis loop. In spite of their low Curie temperature values very high temperature stability of magnetization is possible by suitably adjusting the compensation temperature, which is a unique feature available in garnets containing rare-earth substituents. High power rating is generally obtained in rare-earth garnets but it adversely affects the magnetic loss parameter of the material.

Lithium ferrites have a number of advantages over the other two types of ferrites specially for phase shifter applications. Their main features are: (i) significantly high temperature stability due to high Curie temperatures, unsubstituted lithium ferrite $\left(\mathrm{Li}_{0.5} \mathrm{Fe}_{2.5} \mathrm{O}_{4}\right)$ has the value of $T_{\mathrm{c}}=640^{\circ} \mathrm{C}$, highest among all known ferrites; (ii) higher limit of saturation magnetization up to $5000 \mathrm{G}$ in substituted lithium ferrites gives a distinct advantage for operation at frequencies above $\mathrm{X}$-band region; (iii) higher values of dielectric constant (15 to 20), compared to 10-12 for MgMn ferrite and 14-15 for garnets, enables devices to be fabricated smaller in size and lower weights and (iv) have a low ratio of magnetostriction to magnetocrystalline anisotropy which means low stress sensitivity of remanence.

Several series of lithium ferrites have appeared in the international market. It has been possible to prepare materials of wide ranging properties by introducing metal ions like Ti, Zn, Mn, Co, Ni etc in Li-ferrite compositions (Baba et al 1972). Each type of ion and its quantity as a substituent in the molecular formula unit of lithium ferrite $\left(\mathrm{Li}_{0.5} \mathrm{Fe}_{2.5} \mathrm{O}_{4}\right)$ has a definite role to play in deciding the properties of the material.

Tetravalent titanium when substituted in lithium ferrite lowers the magnetization value and is primarily used for preparing compositions of different $4 \pi M_{\mathrm{s}}$ values below $3750 \mathrm{G}$, which is the value for unsubstituted lithium ferrite. Furthermore, improvement in sintering, control of microstructure and porosity is obtained along with lowering of magnetocrystalline anisotropy. Zinc ions, which predominantly enter the tetrahedral sites of the spinal structure, enhance the $4 \pi M_{s}$ value and is responsible for preparing 
lithium ferrites up to 5000 Gauss. Zinc ions also promote grain growth and densification. It is very effective in lowering the magnetocrystalline anisotropy. However, in large concentrations, it deteriorates the rectangularity of the hysteresis loop. Very low resonance linewidth $(\Delta H)$ values are achievable in $\mathrm{Li}-\mathrm{Zn}$ ferrites.

Substitution of manganese in small content is a well established practice. It inhibits the formation of any $\mathrm{Fe}^{2+}$ ions thus resulting in high resistivity of the material. Another notable effect of $\mathrm{Mn}$ ions is in lowering of stress sensitivity. Presence of a small amount of $\mathrm{Ni}$ ions have been found to be useful in improving the remanence ratio of the material.

For high power devices, as discussed above, it is necessary to increase the power handling capability of the ferrite. Incorporation of fast relaxing ions like divalent Co-ions in very small concentrations has proved highly effective in accelerating spin-lattice relaxation process thereby increasing $\Delta H_{\mathrm{k}}$.

The chemical formula unit of a practical lithium ferrite grade is represented as

$$
\mathrm{Li}_{0 \cdot 5+(t-z-b-c) / 2} \mathrm{Zn}_{z} \mathrm{Ti}_{t} \mathrm{Mn}_{a} \mathrm{Ni}_{b} \mathrm{Co}_{c} \mathrm{Fe}_{2 \cdot 5-(z+3 t+2 a+b+c) / 2} \mathrm{O}_{4}
$$

The substitution levels i.e. values of $z, t, a, b$ and $c$ are varied over limited ranges due to constraints in their accommodation in the spinel structure. To select the composition for a useful material for a specific microwave device a compromise has to be made between the contents of various substituents to achieve the desired results (Pran Kishan 1993a).

In addition to the adjustments in chemical composition the process parameters play an important role in deciding the microstructure and porosity of the sintered material. The grain structure is quite effective in controlling several properties of the material. Use of sintering aid such as low melting $\mathrm{Bi}_{2} \mathrm{O}_{3}$ has become a practice in preparation of lithium ferrite. Addition of $\mathrm{Bi}_{2} \mathrm{O}_{3}$ up to one percent by weight has been found highly useful in lowering the sintering temperature to values where the loss of volatile lithia from the materials is prevented. It is also helpful in grain growth and achieving high density of material (Pran Kishan et al 1985).

The techniques like isostatic pressing. hot pressing, hot-isostatic pressing etc have also emerged as tool for obtaining uniform and high densities along with controlled grain growth. The above routes are often followed in preparing fine grained materials for high power applications.

The work carried out at SSPL on development of substituted lithium ferrites, particularly for phase shifters, is described below.

A number of series of lithium ferrites materials have been developed. These series cover a wide range of properties for application in a variety of components operating at different frequency ranges in the microwave spectrum. These are:

(I) Low power-rectangular loop: It is basically a LiTiZn ferrite family covering $4 \pi M_{\mathrm{s}}$ range up to $5000 \mathrm{G}$ for application up to $\mathrm{mm}$-wave frequency bands.

(II) Wide temperature-rectangular loop: The series covers a $4 \pi M_{\mathrm{s}}$ range up to $3500 \mathrm{G}$ and have comparatively higher Curie temperatures. This LiTi ferrite family is useful for devices requiring stability of its characteristics over a wider range of temperatures.

(III) Low linewidth: A limited range of $4 \pi M_{\mathrm{s}}$ up to $1800 \mathrm{G}$ is covered by the grades of this LiTiZn ferrite family with high zinc concentrations. The main characteristics of this series is the low resonance linewidths (50 to $100 \mathrm{Oe}$ ) and can replace costly garnet materials in some of the components operating below X-band. 
The abovesaid series are fully characterized for all properties of technical importance necessary for design of a microwave device. The reproducibility of the materials has been established and used by agencies like ECIL, ITI and our sister DRDO laboratories in devices like isolators, circulators and attenuators.

Extensive investigational work has been carried out for the development of suitable lithium ferrite grades for use in X-and C-band dual mode phase shifters. Effect of various substituent ions on the properties of the ferrites has been studied in detail. Based on these results appropriate compositions were worked out to obtain materials of desired magnetic and electrical properties. The other important aspect in this connection is the process parameters involved in preparation of the ferrites, i.e. powder preparation, compaction and sintering. In order to control properties which are sensitive to grain structure and density of the sintered material the procedure for compacting of calcined powders into rod shapes and sintering schedules were precisely optimized.

Along with the ferrite material the C-band phase shifter development was also undertaken. The work carried out together on material and device proved to be of immense use in the progress of the activities. The feedback obtained from the experimentation carried out simultaneously on evaluation of ferrite and design of the phase shifter was an important factor in the successful completion of the work.

The specifications of the X-band phase shifter are met by using a grade of the material of LiTi ferrite series possessing highly rectangular hysteresis loop. It has a high Curie temperature of around $500^{\circ} \mathrm{C}$ and $4 \pi M_{\mathrm{s}}$ value of $2250 \mathrm{G}$ and the phase shifters produced with it meet the stringent requirement of its specifications.

The C-band phase shifter is required to operate at moderate power levels of $250 \mathrm{~W}$ peak and $5 \mathrm{~W}$ average. The materials belonging to the above mentioned families of $\mathrm{Li}$ ferrite fail to meet this power rating. Attempts were made to prepare the material by including fast relaxing $\mathrm{Co}^{2+}$ ions in the compositions which is a well known technique used for enhancing power handling capability of the material. Difficulties were experienced since the $\mathrm{Co}^{2+}$ substitution affected the shape of the rectangular hysteresis loop and the magnetic loss factor of the ferrite. Subsequently, ferrite compositions with

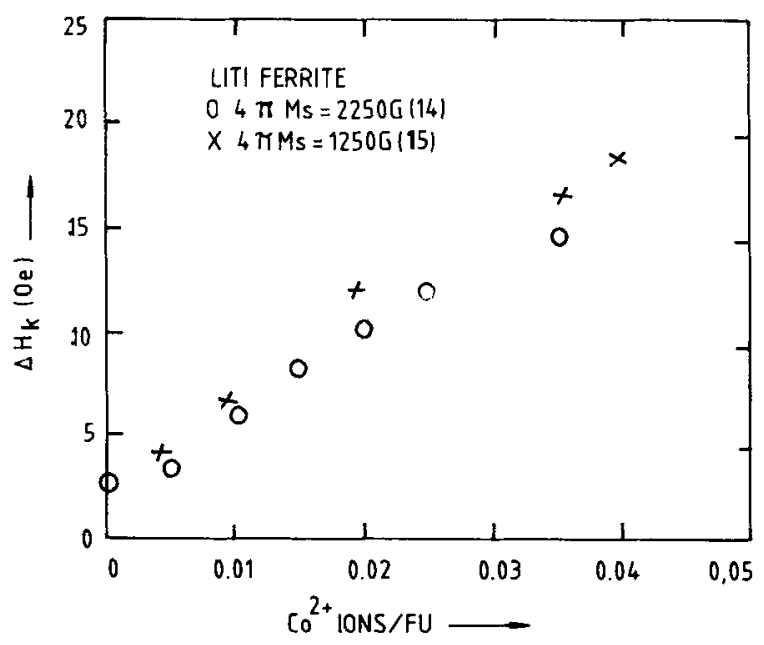

Figure 8. Variation of spinwave linewidth with $\mathrm{Co}^{2+}$ content. 


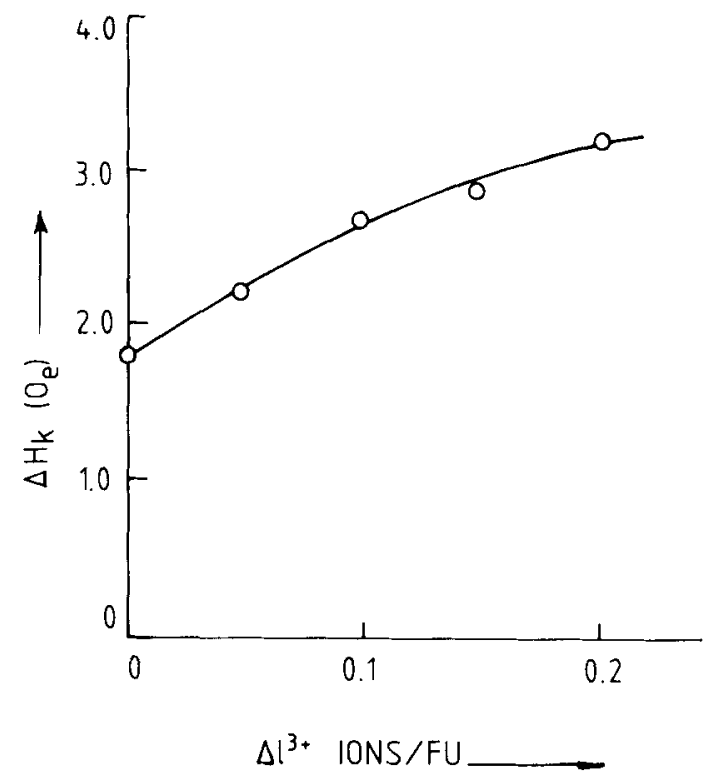

Figure 9. Variation of spinwave linewidth with $\mathrm{Al}^{3+}$ content.

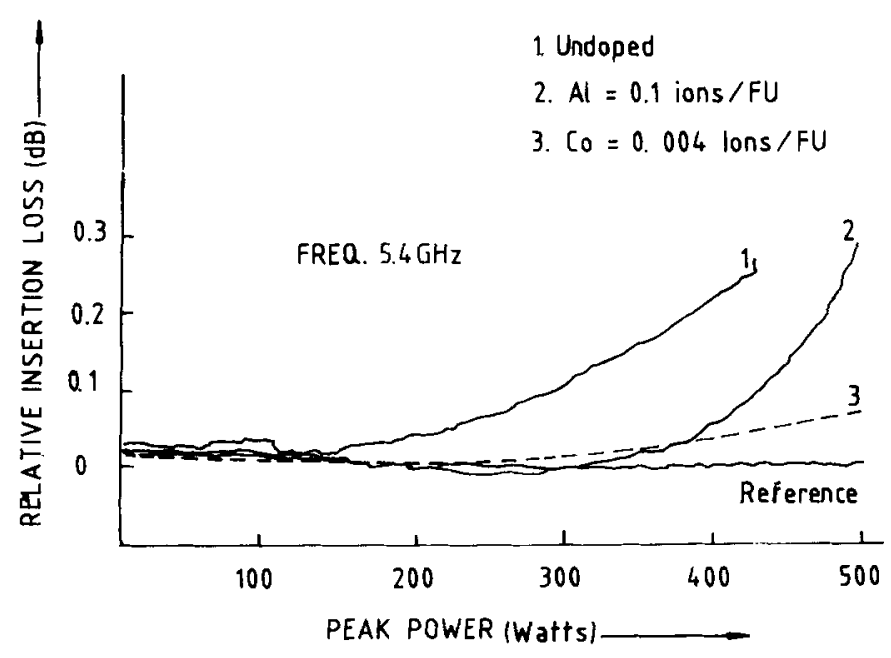

Figure 10. Peak power threshold results for Co and Al substituted LiTi ferrite in dual mode phase shifter configuration.

trivalent aluminium substitution were investigated. $\mathrm{Al}^{3+}$ ions were found to be effective in moderately improving the $\Delta H_{\mathrm{k}}$ values of the material. The effect of $\mathrm{Co}^{2+}$ and $\mathrm{Al}^{3+}$ substitution on $\Delta H_{\mathrm{k}}$ values are shown in figures 8 and 9 (Dionne 1985; Pran Kishan 1993b). It is seen that $\mathrm{Co}^{2+}$ ions even in very small concentration result in sharp increase in $\Delta H_{\mathrm{k}}$ values and for moderate power applications it also becomes difficult to control it precisely during the preparation of the material. On the other hand $\mathrm{Al}^{3+}$ was found to have small but gradual effect on enhancing the $\Delta H_{\mathrm{k}}$ value. Grades of materials belonging to both the types were evaluated in C-band dual mode phase shifter configuration and the observed effect of incident peak power on insertion loss is shown 
in figure $10 . \mathrm{Al}^{3+}$ content of $0 \cdot 1$ ion per formula unit was found to change $\Delta H_{\mathrm{k}}$ from the $1.8 \mathrm{Oe}$ (for $\mathrm{Al}^{3+}=0$ ) to $2.8 \mathrm{Oe}$. This increase was also found to be appropriate for enhancing the peak power threshold of the phase shifter to the required level. The grade of the material found suitable for C-band phase shifters has $4 \pi M_{\mathrm{s}}$ value of $1300 \mathrm{G}$ and $T_{\mathrm{c}}$ of $300 \mathrm{C}$. Other properties of importance like remanence ratio $(R)$, coercive force $\left(H_{\mathrm{c}}\right)$, dielectric contant $(\varepsilon)$, resonance linewidth $(\Delta H)$ of the material are fully characterized

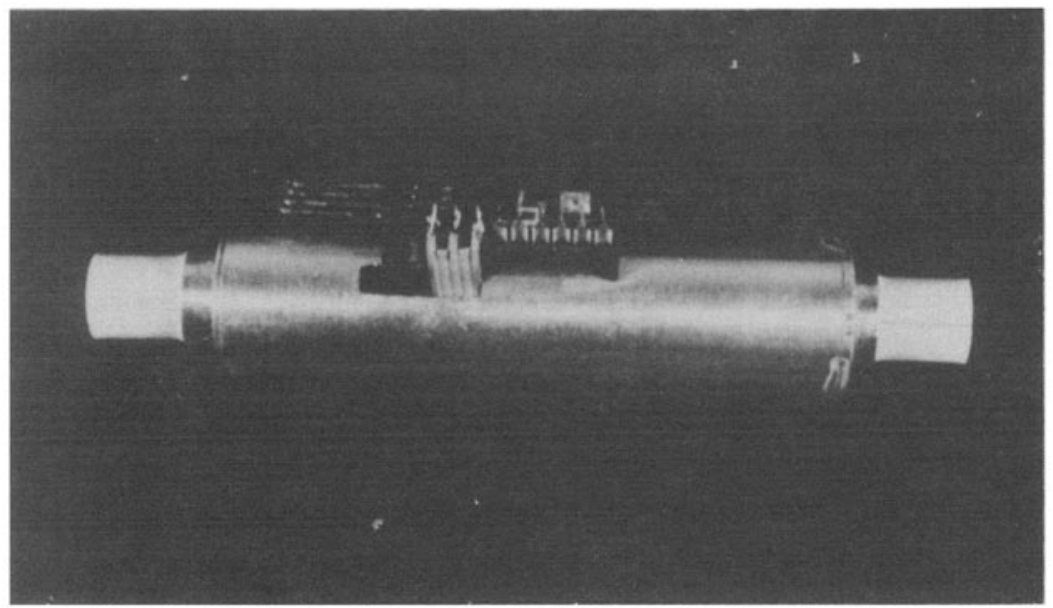

Figure 11. Photograph of a C-band dual mode phase shifter.

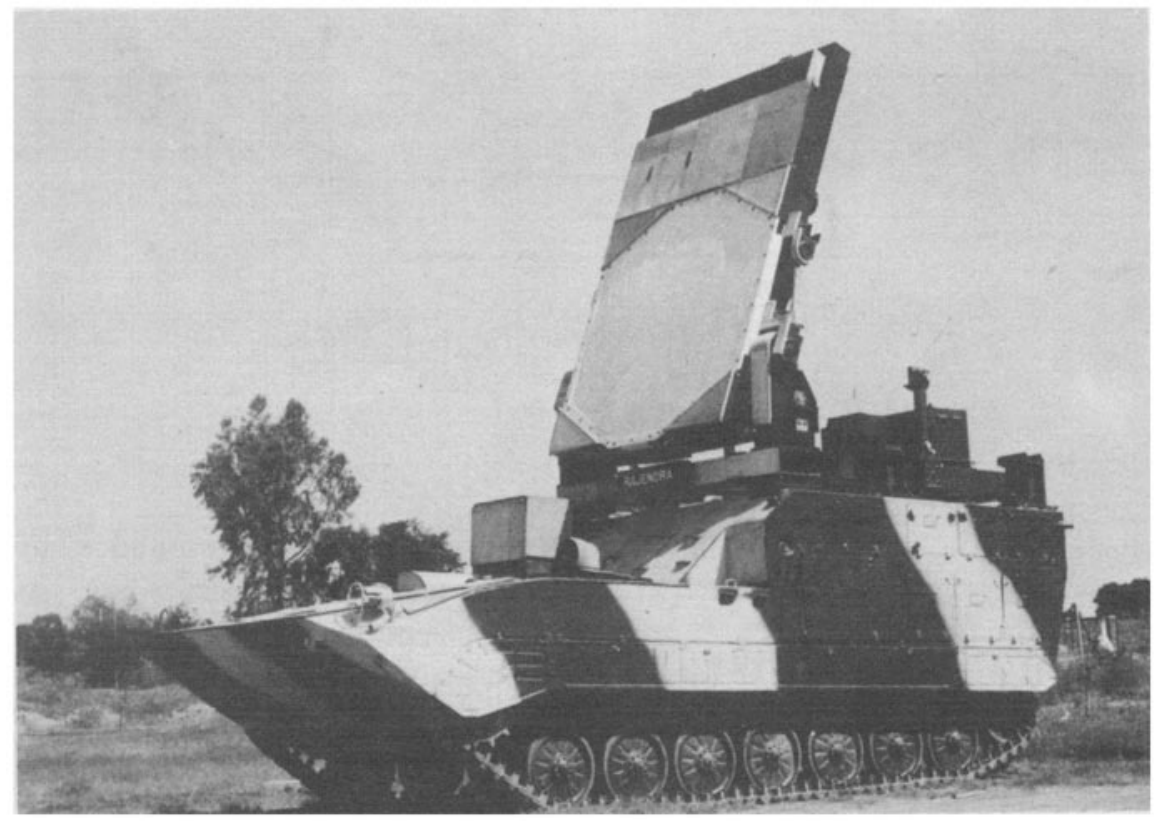

Figure 12. Photograph of Rajendra phased array radar. 
and the phase shifters fabricated with rods of this grade fully meet the desired specifications.

Yokes of both X-and C-band phase shifters are fabricated from a lithium ferrite grade having high values of $4 \pi M_{\mathrm{s}}$ and rectangularity of hysteresis loop (3400 G and $R>0.9$ respectively).

Preparation of ferrites is being carried out by the usual ceramic methods using special procedures for pressing the rods by cold isostatic method. The sintering is carried out in the range $1050-1100^{\circ} \mathrm{C}$. Special emphasis is laid on the microstructure as the grain size and porosity play an extremely important role in controlling the vital properties of materials for its use in phase shifters. The materials are sintered to achieve high densities (better than $96 \%$ of corresponding $X$-ray densities) with an average grain size of more than $50 \mu$.

The ferrite materials developed at SSPL for phase shifters are now being produced by Central Electronics Ltd. (CEL). Production of X- and C-band phase shifters is also being carried out at CEL, based on R\&D work done by IIT, Delhi and SSPL, Delhi. For one multifunction phased array radar, i.e. RAJENDRA, developed by LRDE Bangalore, the requirement of phase shifters is one thousand units of $\mathrm{X}$-band units for missile guidance array and about five thousand units of C-band phase shifters for the main radar. The photographs of the C-band phase shifter and RAJENDRA radar are given in figures 11 and 12.

\section{Conclusions}

With the indigenous development and subsequent productionization of ferrite materials and phase shifters the technology of phased array radar systems has been established in the country. Joint efforts of a consortium of agencies, namely LRDE Bangalore, DRDL Hyderabad, CEL Sahibabad, IIT Delhi and SSPL Delhi has led to this achievement which is considered to be a significant step forward towards self reliance in this strategic area.

\section{Acknowledgement}

The author is thankful to his colleagues of the ferrite group in SSPL for their help.

\section{References}

Bullock C 1982 Military surveillance radar, Intervania 7

Baba P D et al 1972 Trans. IEEE MAG-8 83

Boyd C R Jr 1970 IEEE Trans. MTTT-18 1119

Boyd C R Jr 1976 IEEE MTT-S Int. microwave symp. digest

Boyd C R Jr 1990 Proc. I. Comm-90 429

Curtis D S 1986 Introduction to electronic warfare (Dedham: Artech House)

Dionne G F 1975 Proc. IEEE 63777

Dionne G F 1985 J. Appl. Phys. 573727

Fox A G 1947 Proc. IRE 351489

Koul S K and Bhat B 1991 Microwave and millimeter wave phase shifters (Boston and London: Artech House) Vol. 1 
Pran Kishan 1993a Microwave lithium ferrites, in Microwave materials (eds) V R K Murthy, S Sundaram and B Viswanathan (New Delhi: Narosa Publishing House)

Pran Kishan 1993b High power microwave ferrites, in Recent advances in microwaves (ed.) G P Srivastava (New Delhi: Narosa Publishing House)

Pran Kishan et al 1985 Adv. Ceram. 15207

Rosenbaum F J 1983 Microwave Systems Design Handbook (Palo Alto: EW Communications) p. 153

Truehaft M A and Silber L M 1958 Proc. IRE 481538 